\title{
$d$-wave pairing near a spin-density-wave instability
}

\author{
D. J. Scalapino, E. Loh, Jr., ${ }^{*}$ and J. E. Hirsch ${ }^{\dagger}$ \\ Institute for Theoretical Physics, University of California, Santa Barbara, California 93106
}

(Received 23 June 1986)

\begin{abstract}
We investigate the three-dimensional Hubbard model and show that paramagnon exchange near a spin-density-wave instability gives rise to a strong singlet $d$-wave pairing interaction. For a cubic band the singlet $\left(d_{x^{2}-y^{2}}\right.$ and $\left.d_{3 z^{2}-r^{2}}\right)$ channels are enhanced while the singlet $\left(d_{x y}, d_{x z}, d_{y z}\right)$ and triplet $p$-wave channels are suppressed. A unique feature of this pairing mechanism is its sensitivity to band structure and band filling.
\end{abstract}

Pairing interactions for continuum fermion systems with short-range repulsive interactions have been modeled in terms of paramagnon exchange. ${ }^{1-3}$ Here the bare singleparticle energies are described in terms of an effective mass, and the Fermi surface is spherical. As the strength of the short-range interaction $U$ increases, ferromagnetic correlations are energetically favored, and $s$-wave pairing is suppressed while $p$-wave pairing is enhanced. If the magnetic instability occurs at a finite wave vector, it was recently found that both triplet and singlet pairing are suppressed by backscattering. ${ }^{4}$ However, in a fermion lattice system the band structure and band filling enter in an essential manner, and it is possible to choose a pair wave function which avoids backward scattering. Monte Carlo simulations of the Hubbard model on a lattice and a strong-coupling expansion have suggested pairing in an even-parity anisotropic state. ${ }^{5}$ The purpose of this Rapid Communication is to show, within a paramagnon model on a lattice, that a short-ranged electron-electron repulsion can give rise to even-parity anisotropic pairing near a spin-density-wave (SDW) instability. Emergy has suggested $^{6}$ that this type of mechanism may be responsible for pairing in the Bechgaard salts, and recent experiments ${ }^{7}$ on $\mathrm{UPt}_{3}$ suggest a close relationship between SDW Fermisurface instabilities and superconductivity. As we will show, in a Hubbard model on a cubic lattice a singlet $d$ wave pairing interaction dominates over a wide range of band filling, and the triplet interaction is significant only at very low band filling. ${ }^{8}$

In the Hubbard model, dimensionless coupling constants characterizing the paramagnon mediated pairing interaction in the singlet and triplet channels are obtained from Fermi-surface averages of the zero-frequency singlet

$$
V_{s}=\frac{U^{2} \chi_{0}\left(\mathbf{p}^{\prime}+\mathbf{p}\right)}{1-U \chi_{0}\left(\mathbf{p}^{\prime}+\mathbf{p}\right)}+\frac{U^{3} \chi_{0}^{2}\left(\mathbf{p}^{\prime}-\mathbf{p}\right)}{1-U^{2} \chi_{0}^{2}\left(\mathbf{p}^{\prime}-\mathbf{p}\right)},
$$

and triplet interactions

$$
V_{t}=-\frac{U^{2} \chi_{0}\left(\mathbf{p}^{\prime}-\mathbf{p}\right)}{1-U^{2} \chi_{0}^{2}\left(\mathbf{p}^{\prime}-\mathbf{p}\right)}
$$

illustrated in Fig. 1. Here $\chi_{0}(q)$ is the wave-vector dependent susceptibility of the noninteracting band electrons

$$
\chi_{0}(q)=\sum_{k} \frac{f\left(\epsilon_{k+q}\right)-f\left(\epsilon_{k}\right)}{\epsilon_{k}-\epsilon_{k+q}}
$$

with $\epsilon_{k}=-2 t\left(\cos k_{x}+\cos k_{y}+\cos k_{z}\right)-\mu$ for a cubic lattice. The first term in $V_{s}$ arises from the particle-hole $t$ matrix, while the second term corresponds to the even bubble screening of the interaction. ${ }^{9}$ The triplet interaction $V_{t}$ in the $s= \pm 1$ channels arises from the odd bubble screening of the interaction or equivalently in the $s=0$ channel as the difference between the interactions in Eq. (1).

For a continuum system, one projects the $l=0,2, \ldots$ parts of $V_{s}$ and the $l=1,3, \ldots$ parts of $V_{t}$ to obtain the paramagnon coupling constants for $s, d, \ldots$ and $p, f, \ldots$ pairing, respectively. In this case, as is well known, the ferromagnetic nature of the paramagnons favors triplet $p$-wave pairing. This is also appropriate for a lattice with a small number of electrons in which the Fermi surface is well separated from the Brillouin-zone boundaries and an isotropic effective-mass approximation can be used. However, as more electrons are added to the band and the Fermi surface moves near, or intersects the Brillouin zone, the single-particle energy-band structure modifies the interaction.
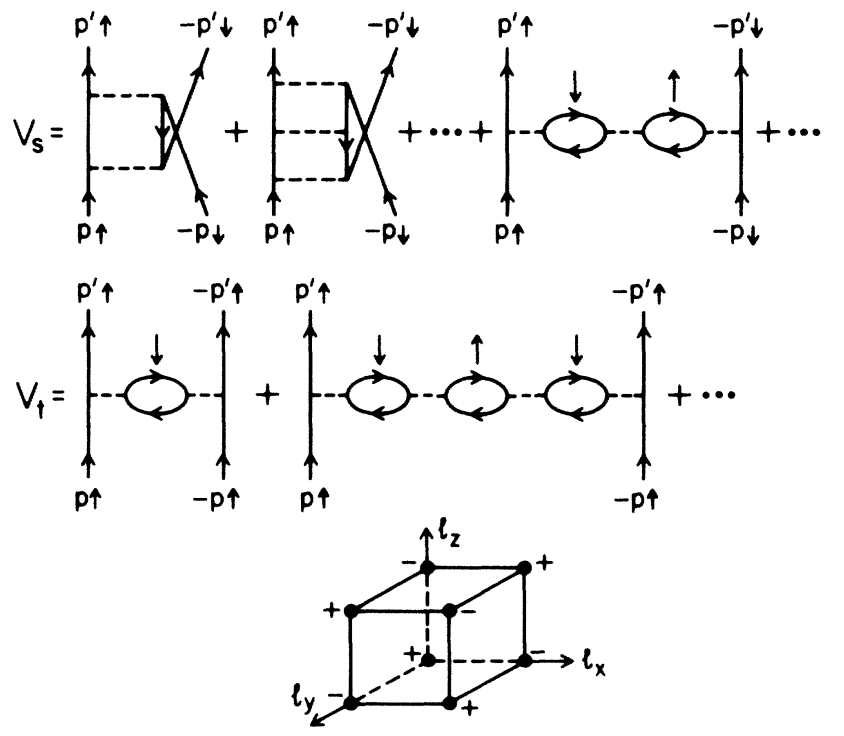

FIG. 1. The paramagnon contributions to the singlet and triplet pairing channels for a Hubbard model. The inset shows the sign of the effective real-space pairing potential for pairs separated by 1 sites on the lattice for $\mu=0$. 
Figures $2(\mathrm{a})-2(\mathrm{c})$ show $\chi_{0}(q)$ plotted along the $[1,1,1]$, $[1,1,0]$, and $[1,0,0]$ directions for different values of $\mu$ at a temperature $\beta=4$. Here we measure energies in units where the one-electron transfer integral $t=1$ (e.g., the bandwidth is 12). For $\mu=0$, the Fermi surface has perfect nesting and exhibits an antiferromagnetic peak at $\mathrm{q}=(\pi, \pi, \pi)$, which diverges logarithmically as the temperature goes to zero. As $\mu$ decreases, this peak becomes finite and shifts to a value of $\mathbf{q}^{*}$, corresponding to the momentum spanning the Fermi surface in the $[1,1,1]$ direction. In this case, as $U$ is increased for a fixed filling, the paramagnon interaction diverges at the spin-densitywave instability, where $U \chi_{0}\left(q^{*}\right)=1$. Finally, for sufficiently small values of $\mu$, the antiferromagnetic peak disappears and $\chi_{0}(q)$ approaches the usual free-electron (Lindhard) behavior. It is in this latter range of fillings that the paramagnon correlations are ferromagnetic and their exchange can give rise to $p$ wave pairing.

For a general filling it is useful to project out $s-, p-$, and $d$-wave coupling constants using simple functions defined on the Fermi surface which are appropriate to a cubic lattice. Thus, for the singlet channel, we define

$$
\bar{\lambda}_{a}=-\int \frac{d^{2} p^{\prime}}{\left|v_{p^{\prime}}\right|} \int \frac{d^{2} p}{\left|v_{p}\right|} g_{\alpha}\left(p^{\prime}\right) V_{s}\left(p^{\prime}, p\right) g_{\alpha}(p) / \int \frac{d^{2} p}{\left|v_{p}\right|} g_{\alpha}^{2}(p) .
$$

Here $p^{\prime}$ and $p$ are integrated over the Fermi surface corresponding to a given band filling set by $\mu$. We have followed the standard practice of putting a minus sign in Eq. (4) so that positive values of the couplings will correspond to an attractive pairing interaction. For the singlet channel, we consider $s$ and $d$ waves. Clearly, for the usual $s$ wave with $g_{0}(p)=1$, the direct Coulomb repulsion will strongly suppress pairing. However, an extended $s$ wave can be constructed which has the form

$$
g_{s} *(p)=A+\left(\cos p_{x}+\cos p_{y}+\cos p_{z}\right) .
$$

If $A=-\mu / 2$, the direct Coulomb interaction will not couple to $g_{s} *$. For $d$-wave pairing we use the $d_{x^{2}-y^{2}}$ and $d_{x y}$ wave functions

$$
g_{x^{2}-y^{2}}=\cos p_{x}-\cos p_{y}, \quad g_{x y}=\sin p_{x} \sin p_{y} .
$$

The other $\Gamma_{3 g}$ channel $g_{3 z^{2}-r^{2}}=2 \cos p_{z}-\cos p_{x}-\cos p_{y}$ has the same coupling as $g_{x^{2}-y^{2}}$, while the other $\Gamma_{5 g}$ channels $\sin p_{x} \sin p_{z}$ and $\sin p_{y} \sin p_{z}$ have the same coupling as $g_{x y}$. For the triplet channel, $V_{s}$ in Eq. (4) is replaced by $V_{t}$ and, for example, the $p_{x}$-wave function is

$$
g_{x}=\sin p_{x} .
$$

Just as in the usual electron-phonon theory, the coupling constants are renormalized. We have estimated this by dividing $\bar{\lambda}_{\alpha}$ by $1+\lambda_{z}$ to take into account the wave function and effective-mass renormalizations. Here $\lambda_{z}$ is given by

$$
\lambda_{z}=\int \frac{d^{2} p^{\prime}}{\left|v_{p^{\prime}}\right|} \int \frac{d^{2} p}{\left|v_{p}\right|}\left(\frac{U^{3} \chi_{0}^{2}\left(p-p^{\prime}\right)}{1-U \chi_{0}\left(p-p^{\prime}\right)}+\frac{U^{2} \chi_{0}\left(p-p^{\prime}\right)}{1-U^{2} \chi_{0}^{2}\left(p-p^{\prime}\right)}\right) / \int \frac{d^{2} p}{\left|v_{p}\right|} .
$$

Thus the effective coupling constant for $\alpha$-wave pairing is

$$
\lambda_{a}=\frac{\bar{\lambda}_{\alpha}}{1+\lambda_{z}} .
$$

Figure 3 shows the effective coupling $\lambda$ for the different pairing configurations versus $\mu$ for $U=4$. In this case, the paramagnon propagator exhibits a spin-density-wave instability when $\mu$ exceeds -0.8 . For $\mu$ values just below

(a)
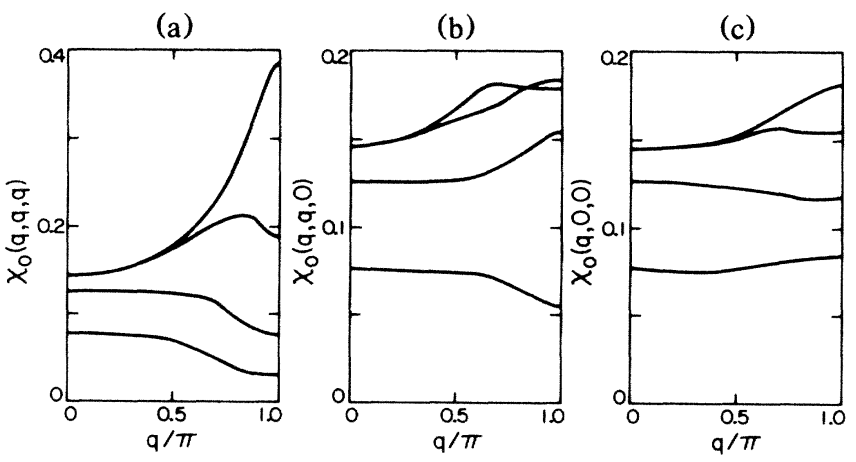

FIG. 2. The susceptibility $\chi_{0}\left(q_{x}, q_{y}, q_{z}\right)$ for a simple cubic band with different fillings plotted along (a) $[1,1,1]$, (b) $[1,1,0]$, and (c) $[1,0,0]$. The curves on each figure, top to bottom, correspond to $\mu=0,-1,-2$, and -3 , respectively. this, the paramagnon interaction strongly favors singlet $d_{x^{2}-y^{2}}$ (or $d_{3 z^{2}-r^{2}}$ ) pairing. The extended $s$-wave coupling, denoted by $s^{*}$ is also attractive near the SDW instability, but it is significantly weaker than the $d_{x^{2}-y^{2}}$ coupling. In this region, the $d_{x y}$ and $p_{x}$ couplings are repulsive. As $\mu$ decreases, moving away from the SDW insta-

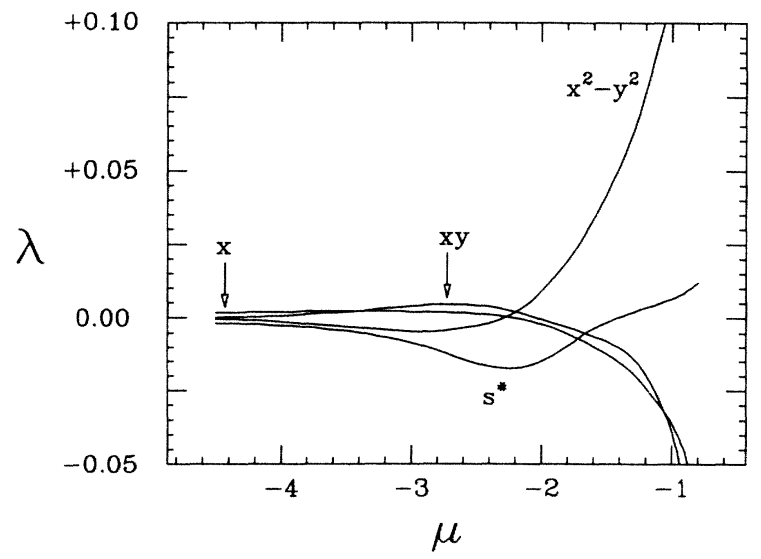

FIG. 3. The effective coupling $\lambda_{a}$ for the $s^{*}, d_{x^{2}-y^{2}}, d_{x y}$, and $p_{x}$-wave channels vs $\mu$ for $U=4$. Here the spin-density-wave instability $U \chi_{0}\left(q^{*}\right)=1$ occurs when $\mu$ exceeds -0.8 . 
bility, the $s^{*}$ and $d_{x^{2}-y^{2}}$ couplings decrease, becoming repulsive, while the $d_{x y}$ and $p_{x}$ couplings become attractive. For $\mu$ slightly below -2.0 , there is a crossover from $d_{x^{2}-y^{2}}$ to $d_{x y}$ pairing. At lower fillings where the paramagnon is ferromagnetic, the $p$-wave channel denoted by $x$ in Fig. 3 is favored. However, in this low filling region with $U=4$, the system is far away from the ferromagnetic instability, so that the $p$-wave attraction is extremely weak.

Although the interactions given by Eqs. (1) and (2) have $\omega=0$, they arise in the Eliashberg ${ }^{10}$ formalism from integrations over $\omega$-dependent spectral weights divided by an energy denominator $\omega$. Their simple real, $\omega=0$, form is just a consequence of the Kramer-Kronig relation. It is useful to keep this in mind, since for values of $\mu$ below the SDW instability the interaction $V_{s}\left(p^{\prime}, p\right)$, Eq. (1), is positive definite. However, its Fourier transform can oscillate and the electrons making up a pair can arrange their space-time correlations to fit into the attractive regions. ${ }^{11}$ To see this in more detail, consider the pairing interaction for $\mu=0$. From Fig. 2 , it is clear that $\chi_{0}$ peaks at the corners of the Brillouin zone. Dynamic correlations enhance and narrow these peaks in the pairing potentials Eqs. (1) and (2). The Fourier transform of $V_{s}$ leads to a real-space pairing potential. For $\mu$ near 0 , this real space pairing potential is approximately given by

$$
\frac{3}{2} \frac{U^{2} \chi_{0}^{*}}{1-U \chi_{0}^{*}} e^{-\left(1^{2} / l_{\gamma}^{2}\right)}\left[\cos \left(\pi l_{x}+\pi l_{y}+\pi l_{z}\right)+\cos \left(\pi l_{x}-\pi l_{y}-\pi l_{z}\right)+\cos \left(\pi l_{x}+\pi l_{y}-\pi l_{z}\right)+\cos \left(\pi l_{x}-\pi l_{y}+\pi l_{z}\right)\right],
$$

where $l_{0}^{2} \sim\left(1-U \chi_{0}^{*}\right)^{-1}$ with $\chi_{0}^{*}=\chi_{0}(\pi, \pi, \pi)$. The staggered structure of this potential is illustrated in the inset of Fig. 1. It is repulsive for zero pair separation $1=0$, attractive for a near-neighbor separation, repulsive for a nextnear-neighbor separation, etc. It is clearly attractive for $\left(d_{x^{2}-y^{2}}, d_{3 z^{2}-r^{2}}\right)$ pairing, less so for $s^{*}$ pairing, and repulsive for $\left(d_{x y}, d_{y z}, d_{x z}\right)$ pairing.

Now, just as for ${ }^{3} \mathrm{He}$, the simple paramagnon theory cannot provide definitive results. Rather it suggests possible phenomenological relations. In particular, the occurrence of a strongly enhanced singlet $d$-wave coupling mediated by spin fluctuations near an SDW instability provides a framework for thinking about some of the heavy-fermion superconductors such as $\mathrm{UPt}_{3}$ and the Bechgaard salts ditetramethyltetraselenafulvalene $X$. The strong interplay of band structure, band filling, and many-body effects appears in a natural manner reflecting the role of Fermi surface nesting on the pairing interactions. The effect of pressure can clearly alter the oneelectron transfer matrix elements which directly affect the band structure and this nesting. Once the band structure and $\mu$ are fixed, one coupling constant $U$ determines both the SDW and pairing responses. This unified theory should provide important constraints for the competition and possible coexistence of the SDW and pairing phases. ${ }^{12}$

We would like to acknowledge useful discussions with J. W. Wilkins. This work was supported in part by the National Science Foundation under Grant No. PHY8217853, supplemented by funds from the National Aeronautics and Space Administration. We also acknowledge National Science Foundation support under Grants No. DMR83-20481 and No. DMR84-51899.
*Present address: Los Alamos National Laboratory, P. O. Box 1663, Los Alamos, NM 97545.

†Permanent address: Department of Physics, University of California, San Diego, CA 92093.

${ }^{1}$ N. F. Berk and J. R. Schrieffer, Phys. Rev. Lett. 17, 433 (1966).

2P. W. Anderson and W. F. Brinkman, Phys. Rev. Lett. 30, 1108 (1973).

${ }^{3}$ K. Levin and O. T. Valls, Phys. Rep. 98, 1 (1983).

${ }^{4}$ M. T. Beal-Monod, C. Bourbonnais, and V. J. Emery (unpublished).

5J. E. Hirsch, Phys. Rev. Lett. 54, 1317 (1985).

${ }^{6}$ V. J. Emery, Synth. Met. 13, 21 (1986); J. Phys. (Paris) Colloq. 44, C3-977 (1983).

${ }^{7}$ G. Aeppli, E. Bucher, and G. Shirane (unpublished); A. P. Ramirez, B. Batlogg, E. Bucher, and A. S. Cooper (unpublished).
${ }^{8}$ The variation of the $p$-wave coupling with band filling in the paramagnon model was discussed by D. J. Scalapino, in Condensed Matter Physics: The Theodore D. Holstein Symposium, 1986, edited by R. L. Orbach (Springer, New York, 1986).

${ }^{9}$ The direct Coulomb interaction gives a repulsive contribution to $V_{s}$, but because of its flat frequency response it is usually treated by a pseudopotential approximation, see Ref. 1 .

${ }^{10}$ G. M. Eliashberg, Zh. Eksp. Teor. Fiz. 38, 726 (1960) [Sov. Phys. JETP 11, 696 (1960)].

${ }^{11}$ W. Kohn and J. M. Luttinger, Phys. Rev. Lett. 15, 524 (1965).

${ }^{12}$ In previous studies of the competition between the SDW and superconducting phases the SDW instability was driven by $U$, and the $s$-wave pairing instability was driven by an independent electron-phonon coupling $g$. See, for example, K. Machida, J. Phys. Soc. Jpn. 50, 2195 (1981). 